\title{
EGG VOLUME AND SURFACE AREA CALCULATIONS BASED ON MACHINE VISION
}

\author{
Ping Zhou, Wengang Zheng ${ }^{*}$, Chunjiang Zhao, Changjun Shen, Gang Sun \\ National Engineering Research Center for Information Technology in Agriculture, Beijing, \\ China, 100097 \\ * Corresponding author, Address: Room 507, Building A, Beijing NongKe Masion, 11\# \\ Shuguang Huayuan Middle Road, Haidian District, Beijing, China,100097,Tel:+86-10- \\ 51503590,Email: zhengwg@nercita.org.cn
}

\begin{abstract}
Egg volume $(V)$ and surface area $(S)$ are two important geometrical calculations for the poultry industry and in biological studies, as they can be used in research on population and ecological morphology, and to predict chick weight, egg hatchability, shell quality characteristics, and egg interior parameters. With machine vision technology, the objective of the research herein was to develop a much more fast and available method to predict them. Besides, the researcher gave the definition of Volume in Pixels $\left(V_{p}\right)$ and Surface area in Pixels $\left(S_{p}\right)$ for the first time. Finally, new linear models were established. The $R$ value for volume model is 0.88 and surface area is 0.86 . The prediction ability of the models are: for volume, the correctness reaches $52 \%$ with $\pm 1 \mathrm{~cm}^{3}$ statistical errors, $89 \%$ with $\pm 2 \mathrm{~cm}^{3}$, for surface, the correctness reaches $49 \%$ with $\pm 1 \mathrm{~cm}^{2}$ statistical errors, $87 \%$ with $\pm 2 \mathrm{~cm}^{2}$.
\end{abstract}

Keyword: Egg, Volume,Surface area, Machine vision

\section{INTRODUCTION}

Egg volume and surface area are important geometrical calculations in poultry studies, which were reported by Narushin(2001b,2002,2002a,2002b). So how to measure them quickly and accurately is considered to be a hot topic. As far as so on, several models, based on the measurements of the egg length $(L)$ and the maximum breadth $(B)$, have been suggested to calculate the parameters. The basic formula for calculating egg volume was:

$$
V=k_{v} L B^{2},
$$

Please use the following format when citing this chapter:

Zhou, P., Zheng, W., Zhao, C., Shen, C. and Sun, G., 2009, in IFIP International Federation for Information Processing, Volume 295, Computer and Computing Technologies in Agriculture II, Volume 3, eds. D. Li, Z. Chunjiang, (Boston: Springer), pp. 1647-1653. 
in which $k_{v}$ is a coefficient for volume calculation. The coefficient values were firstly reported by Romanoff(1949). Later, data gave $k_{v}=0.523$ (Ayupov,1976), and $k_{v}=0.496$ (Narushin, 1997a), and a number of values of $k_{v}$ were proposed by Hoyt(1979). Narushin(2001a) showed that egg volume can also be estimated by means of a theoretically deduced formula:

$$
V=\frac{2 \pi L^{3}}{3(3 n+1)}
$$

in which $n=1.057\left(\frac{L}{B}\right)^{2.732}$.

Later, Narushin(2005) gave a more accurate and available formula than ever before:

$$
V=(0.6057-0.0018 B) L B^{2}
$$

Similar to equation(1) and taking into account the changes of the dimensionalities, the lasted formula for calculating $S$ were reported by Narushin(2005):

$$
S=(3.155-0.0136 L+0.0115 B) L B
$$

However, there are still several problems: firstly, the supposed curve in the reported models( taking Narushin,1997's for example) will not always best resemble all eggs' shapes; secondly, if thus, the measurements of $L$ and $B$ with a vernier caliper can't be fast and automatic, which will not be acceptable in poultry industry.

Thus, with machine vision technology, the objective of the research herein was to develop a much more fast and available method to solve the above problems. Characteristic parameters firstly defined by the researcher were calculated from the edge information detected from the egg image. Besides, new linear models were built to predict the volume and surface area based on the calculated parameters.

\section{MATERIAL AND METHODS}

\section{1 $\quad$ Egg samples}

In this experiment, 100 fresh eggs were collected from hens of 70 weeks old raised by Hubei Xiantao Food Corporation in China. They were labeled in order after cleaning and air-drying. 


\subsection{Experimental system}

As shown in Fig.1, the test system was composed of lamp(DPS-24W304T), CCD camera(SRC-500HP), Additional Lamp, Image Card(DH-VRTCG200), Computer, and etc.

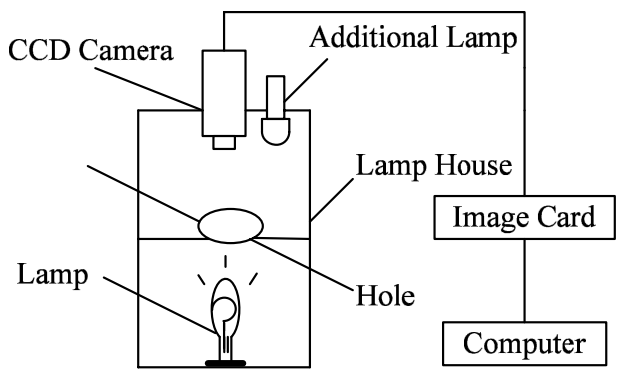

Fig.1 the test system

\subsection{Experimental Method}

\subsubsection{Specific procedure}

The experiment should be excuted through these steps: (i) put each egg on the hole, through where the light can shine out, and (ii) adjust the direction of egg till the projection of longitudinal axis in the image was vertical in the screen(manual viewing the screen while adjusting), then capture the image and store it in the harddisk, and (iii) with the selfdeveloped programme, detect the edge information, later, calculate the characteristic parameters of volume in $\operatorname{pixels}\left(V_{p}\right.$, unit: $p_{i x}{ }^{3}, p_{i x}=$ pixel $)$ and surface area in pixels $\left(S_{p}\right.$, unit: $\left.p_{i x}{ }^{2}\right)$, which were explained in the next paragraph, and (iv) calculate the exact volume $V$ and $V_{s}$ using the difference between the measurements of egg weight in air and water, and finally calculate the exact surface area $S$ from the formula:

$$
S=V_{s} / T_{s}
$$

where $V_{s}$ is the volume of the eggshell, $T_{s}$ the average thickness of the eggshell, measured by vernier caliper.

\subsubsection{The algorithm for characteristic parameters}

As practice shows, if en egg may be considered as a solid of revolution about the longitudinal axis, it is possible to consider the contour of the cross 
section to be circular. Fig. 2 was the profile of an egg, $L$ is the longitudinal axis, $O$ is the peak, Line $M N$ is perpendicular to longitudinal axis, $z$ is the perpendicular distance from the cross section to the peak of the profile, $M$ is the left intersection with the profile, and $N$ is the right, $\theta(z)$ is the angel between long axis and the tangent at point $M$. Fig. 3 is the cross section at the position of Line $M N, A(z)$ is the area and $C(z)$ is the circumference length.

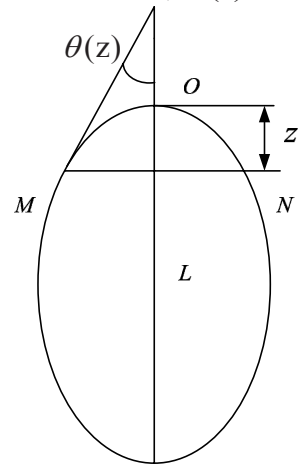

Fig.2 The profile of egg

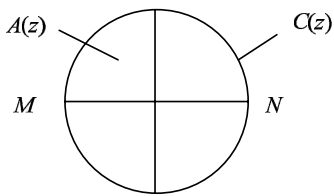

Fig.3 $M N$ cross section

Hence, from the perspective of the differential calculus and integral calculus, the volume $V$ and surface area $S$ of the solid of revolution about the long axis can be found from the equations

$$
V=\int_{L} A(z) d z
$$

and

$$
S=\int_{S^{\prime}} C(z) d s
$$

Where $s^{\prime}$ is the left contour of the egg's half-projection, $d s$ is the differential operator along $s^{\prime}$, with the following relation to $d z$.

$$
d s=d z / \cos \theta(z)
$$

Thus, equation (7) can be rewritten as

$$
S=\int_{L} \frac{C(z)}{\cos \theta(z)} d z
$$

Egg edge found out from the image was similar to the profile shown in Fig.2. Considering the discrete edge information, Volume in pixels $\left(V_{p}\right)$ and Surface in pixels $\left(S_{p}\right)$ are defined as followings: 


$$
V_{p}=\sum_{i=0}^{L} A\left(z_{i}\right)
$$

and

$$
S_{p}=\sum_{i=0}^{L} \frac{C\left(z_{i}\right)}{\cos \theta\left(z_{i}\right)}
$$

Where $L$ (unit: $p_{i x}$ ) is long axis in the image, $A\left(z_{i}\right)={ }^{\pi d^{2}\left(z_{i}\right) / 4}, d\left(z_{i}\right)$ (unit: $\left.p_{i x}\right)$ is the diameter of the cross section which has a distance of $z_{i}$ to the peak of the edge in the image, $C\left(z_{i}\right)$ is the corresponding circumference length, $\theta\left(z_{i}\right)$ has the similar meaning to the above $\theta(z)$, which can also be calculated from the edge information.

\section{RESULTS AND DISCUSSION}

The results of the measurements and calculations are shown in Fig.4 and Fig.5. Fig.4 is the scattergram of samples about the $V$ and $V_{p}$, from which we can see that the volume $(V)$ seems to increase linearly accompanying with the increasing of $V_{p}$. Hence, with SAS V9.0, we can easily find the model to predict egg volume:

$$
V=3.447 E-05 \times V_{p}+23.296991
$$

with $R=0.88$. The proportion of samples with statistical errors in domain of $\pm 1 \mathrm{~cm}^{3}$ reaches $52 \%$, and $89 \%$ in $\pm 2 \mathrm{~cm}^{3}$.

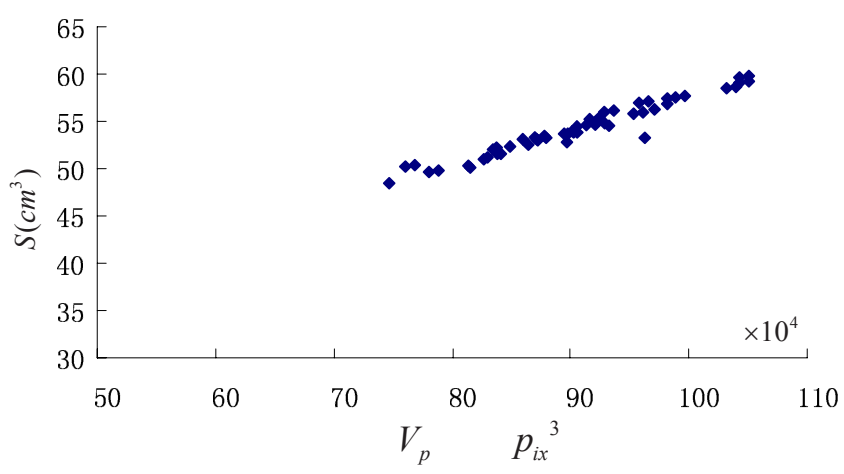

Fig.4 Scattergram of samples about $V$ and $V_{p}$ 
And Fig.5 is the scattergram of samples about $S_{p}$ and $S$, from which we can see that the surface area $(S)$ tends to become large as long as the increasing of $S_{p}$ linearly. Thus, we can easily get the model for surface area:

$$
S=5.985 E-04 \times S_{p}+44.684279
$$

with $R=0.86$. The proportion of samples with statistical errors in domain of $\pm 1 \mathrm{~cm}^{2}$ reaches $49 \%$, and $87 \%$ in $\pm 2 \mathrm{~cm}^{2}$.

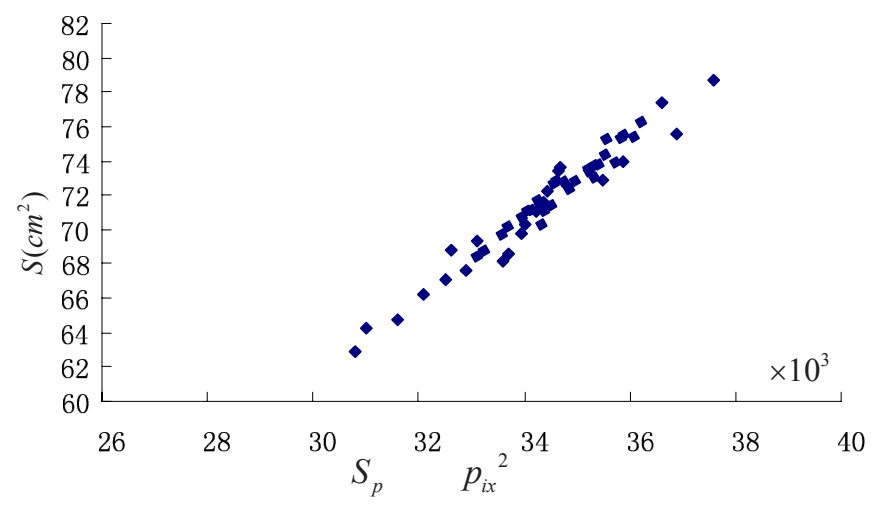

Fig.5 Scattergram of samples about $S$ and $S_{p}$

There are may several factors working on the models' precision: (1) the projection of egg's longitudinal axis in the image may not be strictly vertical,(2) the machine vision system may have dimension distortion. However, the results show that machine vision technology can also be used to predict volume and surface area instead of traditional models in practice. Besides, the new method can improve the calculation efficiency, which will be welcome in poultry industry and biological studies. Furthermore, the linear models can be explained much better in geometrics.

\section{ACKNOWLEDGEMENTS}

This study has been funded by China National 863 Plans Projects (Contract Number: 2006AA10Z202)

\section{REFERENCES}

Ayupov, F. G. 1976. On the egg mathematical model. Advanced Scientific-Industrial Experience in Poultry Breeding. Express Information. 9:14-16.(In Russian) 
Hoyt, D.W. 1979. Practical methods of estimating volume and fresh weight of bird eggs. AuK96:73-77.

Narushin, V. G. 1997a. The avian egg: Geometrical description and calculation of parameters. J. Agric. Eng. Res. 68:201-205.

Narushin, V. G. 2001a. Shape geometry of the avian egg. J. Agric. Eng. Res. 79:441-448.

Narushin, V. G. 2001b. What egg parameters predict best its shell strength? Pages 349-355 in IX European Symposium on the Quality of Eggs and Egg products. Kusadasi, Turkey.

Narushin, V. G. 2005. Egg Geometry Calculation Using the Measurements of Length and Breadth. Poultry SCI. J. 84:482-484.

Narushin, V. G., and M. N. Romanov 2002a. Physical characteristics of chicken eggs in relation to their hatchability and chick weight. Paper \#026066 in CD-ROM Proceedings of ASAE Annual International Meeting/CIGR World Congress, Chicago, IL.

Narushin, V. G., and M.N. Romanov. 2002b. Egg physical characteristics and hatchability. World Poult. SCI. J. 58:297-303.

Narushin, V. G., M. N. Romanov, and V. P. Bogatyr. 2002. Relationship between preincubation egg parameters and chick weight after hatching in layer breeds. Biosystems Eng. 83:373-381.

Romanoff, A.L., and A.J.Romanoff. 1949. The Avian Egg. John Wiley, New York, NY. 